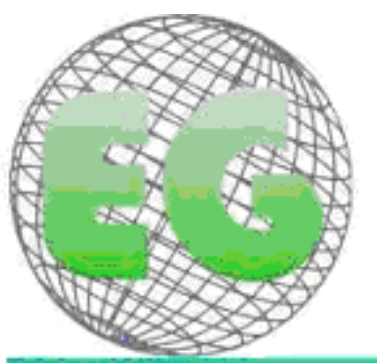

15SN 1696-6*\$:

\title{
RELACIÓN TERAPÉUTICA DEL PERSONAL DE ENFERMERÍA CON LOS PADRES DE NEONATOS EN LA UNIDAD TERAPIA INTENSIVA NEONATAL
}

NURSING PERSONNEL'S THERAPEUTIC RELATIONSHIP WITH THE PARENTS OF NEWBORNS IN THE NEWBORN INTENSIVE CARE UNIT

* Sifuentes Contreras, A., Parra, FM., Marquina Volcanes, M., Oviedo Soto, S.

*Lcda. en Enfermería. Profesora Universidad de los Andes. Venezuela.

Palabras-clave: Enfermería, Relación Terapéutica; Recién Nacidos, Padres, UCIN

Key words: Nursing, Therapeutic Relationship, Newborns, Parents, and NICU.

\section{RESUMEN}

El objetivo fue determinar las características de la relación terapéutica que establece el Personal de Enfermería con los padres de los neonatos hospitalizados en la Unidad de Terapia Intensiva Neonatal (UTIN), del Hospital Central de San Cristóbal. Táchira, Venezuela. El estudio fue descriptivo, con diseño transversal, con una población de 30 padres, a quienes se les aplicó un cuestionario de 19 ítems. El análisis e interpretación de los resultados se realizó con base a estadística descriptiva. En la dimensión comunicación, los padres opinaron que el Personal de Enfermería no se presentó ni les dio a conocer su nombre; tampoco se dirigieron a ellos por su nombre; no le explicaron sobre el tratamiento que su hijo recibía, qué exámenes se les realizaron y los cuidados que deberían ser cumplidos en el hogar una vez que sean egresados. Refirieron que el Personal de Enfermería no les dedicó tiempo para conversar. Sin embargo, destacó un alto porcentaje de respuestas positivas en cuanto a que el Personal de Enfermería les recibió de forma amistosa cuando ingresaron a visitar a sus hijos, le aclararon duda y que cumplen con los cuidados de enfermería que el neonato necesita. En la dimensión apoyo emocional, se determinó que los padres recibieron ánimo del Personal de Enfermería y, les brindaron fe y esperanza en cuanto a la recuperación de sus hijos, les respetaron sus condiciones sociales, sus creencias y costumbres culturales, les brindaron apoyo cuando ellos se sintieron preocupados. Consideraron a este personal como digno de confianza y respeto, pero con escasa manifestaciones de afecto en cuanto al contacto físico. 


\section{ABSTRACT}

The aim was to determine the characteristics of the therapeutic relationship established between nursing personnel and the parents of hospitalized newborns in the Newborn Intensive Care Unit, Central Hospital of San Cristóbal, Táchira, Venezuela. The study was descriptive, with cross-sectional design and a sample of 30 parents, to whom were given a 19 item questionnaire. The analysis and interpretation of the results were made based on descriptive statistic. In the communication dimension, the parents answered that the nursing personnel did not introduce themselves or say their names, nor did they call them by their names; or explain the treatment that their newborn received, what tests were made and the care that should be done once they were taken home. They reported that nursing personnel did not take time to talk to them. Nevertheless, they emphasized a high percentage of positive answers such as that nursing personnel greeted them in a friendly way when they arrived to visit their newborns, clarified doubts and fulfilled all the nursing cares that the newborn needed. In the emotional support dimension, it was determined that parents were cheered up by the nursing personnel and gave them faith and hope of the recovery for their children, were respectful of their social conditions, their beliefs and cultural customs and offered support when they were worried. They considered this personnel worthy of confidence and respect, but with low manifestations of affection and physical contact.

\section{INTRODUCCIÓN}

A lo largo de la trayectoria de la disciplina de enfermería, la relación persona - enfermera(o) aparece como una constante tanto en la literatura como en las publicaciones científicas. Así están las grandes teóricas de la profesión que definen Enfermería como una relación que ayuda, que acompaña a la persona, dentro de un entorno a vivir experiencias de salud. Pero en el ámbito práctico, del ejercicio profesional, la relación terapéutica es escasa, lo que se agrava en el hospital por la separación con el entorno propio. Por lo tanto, una relación implica contacto, lazos, unión entre dos o más personas, las cuales establecen un vínculo que los mantiene unidos en un tiempo, en un espacio en un intento por restablecer un equilibrio que les permita vivir de una manera integral o plena. En tal sentido, se puede decir que el hombre es un ser grupal y la manera de establecer vínculos dentro del grupo es a través de la comunicación permitiéndose estar conectados entre sí.

Al respecto, Satir ${ }^{(1)}$ plantea que "La comunicación afectiva es relevante en las interacciones humanas, en unas ocasiones evita situaciones problemáticas y en otras las resuelve". El Personal de Enfermería interactúa con las personas que se encuentran en su entorno con el propósito de identificar sus necesidades interferidas y para conocer también sus sentimientos y sus opiniones acerca de su problema de salud. Es importante que el paciente y los familiares sean partícipes en la búsqueda de soluciones para lograr la calidad de su cuidado. Por lo tanto, la enfermería puede ser considerada como un instrumento educativo, que promueve una fuerza de maduración de la personalidad hacia la construcción de una vida creativa, constructiva, productiva, personal y comunitaria ${ }^{(2)}$.

En concordancia con lo anteriormente señalado, en el ámbito hospitalario la comunicación juega un papel importante ya que a través de una completa y oportuna anamnesis se puede establecer una relación terapéutica (enfermero/usuario) la cual es la que indica la receptividad por parte del usuario en cuanto a la atención proporcionada, establece y mantiene relaciones de apoyo que evita que el usuario en cualquier situación determinada pueda experimentar respuestas negativas, insuficientes e insatisfactorias. Dichas respuestas negativas pueden ser sentimientos de inquietud ante una amenaza imprecisa e inespecífica. Además la relación enfermero/usuario es la que se establece en el ambiente hospitalario y se considera una relación interpersonal de tipo profesional que tiene como fundamento 
identificar las necesidades y/o problemas los cuales limitan el funcionamiento físico y emocional del usuario e indagar sobre las posibles soluciones a sus requerimientos.

Desde esta perspectiva es importante considerar que cuando nace un niño prematuro, se interrumpe el proceso de "anidación psicobiológica"(3) de los padres y el recién nacido, ya que ambos necesitan el tiempo de los nueve meses de gestación, para crear un lugar físico y psíquico para ese nuevo integrante de la familia, lo cual implica una reorganización de su rol. El recién nacido también necesita los nueve meses para madurar biológicamente y encontrarse en condiciones de poder sobrevivir fuera del útero. Por lo tanto, el nacer antes de este período, encuentra tanto a los padres como al bebé no preparados y se crea un estado de vulnerabilidad somática y psíquica en el niño, la madre y el padre.

Este puede agravarse por la situación del niño en una unidad intensiva de alta complejidad, presentándose en ellos una crisis que "implica una perturbación cuyos efectos no pueden ser absorbidos por la propia organización familiar, produciéndose entonces síntomas en sus miembros y la producción de estructuras innovativas que significan una transformación de los modos de funcionamiento"(4). Durante este momento, se ponen a prueba los mecanismos y estrategias que dispone la familia para afrontar situaciones que distan de ser lo esperado en una situación normal como el embarazo y el parto, y que se transforma en una experiencia dolorosa y traumática cuando su recién nacido no es llevado con ellos a compartir el nido del nacimiento, sumada a la interrupción del proceso de vinculación afectiva entre madre-padre y recién nacido, lo cual representa una condición que afecta los procesos familiares en los padres con recién nacidos enfermos y prematuros ${ }^{(5,6)}$.

Los avances en el cuidado de los recién nacidos prematuros han significado un progreso notable en la neonatología moderna. Las unidades de cuidados intensivos neonatales (UCIN) cada vez más eficientes cumplen un papel importante en la disminución de la mortalidad neonatal e infantil ${ }^{(7,8)}$. Sin embargo, como era de esperar, estos resultados alentadores de las UCIN han provocado algunos efectos no deseados, en especial sobre los padres. La experiencia demuestra que la hospitalización en la UCIN provoca reacciones diversas en los padres, en general intensas y perturbadoras ${ }^{(9)}$. El estrés y la depresión de padres y madres son algunos de los aspectos más frecuentemente observados y que pueden llegar a interferir en la organización de la interacción entre ellos y su hijo, como así también en la comunicación con los profesionales que asisten al niño. Los padres que se encuentran en la UCIN, pueden llegar a presentar síntomas físicos de estrés ${ }^{(3)}$ como consecuencia de la presencia de algunos factores, como son: la condición de salud del neonato, las características ambiéntales del lugar en donde se encuentra hospitalizado y la separación del binomio padres- hijo ${ }^{(10)}$.

De allí surge la necesidad de asistir de manera integral a los padres de los recién nacidos hospitalizados en la UCIN, por medio de la relación terapéutica efectiva en la cual se abren mayores expectativas de vida, junto con la preocupación de mantener con vida a este pequeño ser, el profesional de enfermería debe, desde el momento que el RN se hospitaliza, intentar incorporar al niño que nace con problemas a su familia, estimulando la visita de sus padres, promoviendo la manutención de la lactancia materna, tratando de incorporar a los padres al cuidado de su hijo informando su evolución y estado de salud, para aminorar la angustia y desesperanza, así como fomentar los lazos afectivos que se mantendrán durante toda la vida.

El objetivo de este estudio es determinar las características de la relación terapéutica, bajo las dimensiones apoyo emocional y comunicación, que establece el Personal de Enfermería con los padres de los neonatos hospitalizados en la Unidad de Terapia Intensiva Neonatal, del Hospital Central de San Cristóbal. 


\section{PERSONAS Y MÉTODOS}

De acuerdo a los objetivos propuestos y a la naturaleza de la presente investigación, la misma se consideró un estudio descriptivo de naturaleza cuantitativa; con un diseño transversal. La población de estudio estuvo constituida por 30 padres de los neonatos que fueron hospitalizados en la UTIN. Las entrevistas fueron realizadas a la semana de haber ingresado el niño o niña al servicio, después de las visitas programadas y a conveniencia del tiempo disponible por los padres, previo consentimiento informado y siguiendo lo establecido por el Artículo 42 de Código Deontológico de Enfermería, con respecto al la confidencialidad de los datos. El instrumento diseñado fue validado por juicio de expertos, para la evaluación de su contenido. El mismo quedó conformado por un total de 19 ítems que contemplan cada uno de los indicadores propuestos para cada dimensión, con preguntas de opinión sobre la presencia o no de conductas relacionadas con la comunicación y el apoyo emocional que proporciona el profesional a los padres durante la hospitalización del neonato. Una vez aplicado el instrumento y tabulados manualmente con el uso de estadística descriptiva, los datos fueron analizados a partir de las frecuencias absolutas y porcentuales de los mismos.

\section{RESULTADOS}

En la dimensión Comunicación, se determinó que el 86,66\% del Personal de Enfermería, recibió de forma amistosa a los padres de los neonatos hospitalizados cuando visitaron a sus hijos. Los padres opinaron en un 73,33\%, que el Personal de Enfermería no se presentó ni dio a conocer su nombre para identificarse con ellos. Se verificó en un $86,66 \%$ que Personal de Enfermería cuando se dirige a los padres, lo hace por el nombre del neonato y no por el de ellos. Un 73,33 están informados del horario de las visitas; pero el $70 \%$ de la población estudiada no son notificados por el Personal de Enfermería acerca del tratamiento. El $66,66 \%$ de los padres opinó que no le explicaron los exámenes que se les realizaban a sus hijos. El 63,33\% manifestó que no fue orientado acerca del cuidado que necesita su hijo en cuanto a su enfermedad. Con respecto a si el personal de enfermería acude a usted cuando necesita información de su hijo, el 53,33\% expresó afirmativamente. Por otra parte, se encontró en un $56,66 \%$ de las respuestas emitidas por los padres encuestados que no se les orientó sobre los cuidados en el hogar luego del egreso de sus hijos. El $66,66 \%$ de los padres opinaron que el Personal de Enfermería no le dedicó tiempo para conversar sobre la evolución que ha tenido su hijo.

Con respecto a la dimensión Apoyo Emocional, el 53,33\% de los padres opinaron que el Personal de Enfermería no ofreció manifestaciones de afecto a través del contacto físico, el $70 \%$ de los padres indicaron que el Personal de Enfermería síi se preocupaba por cuidar la salud de los recién nacidos allí hospitalizados. Por otra parte, el 53,33\% manifestó que el Personal de Enfermería no les ofreció apoyo cuando se sentían preocupados por sus hijos. El 66,66\% de los padres señalaron que sintieron confianza en el Personal de Enfermería. El $60 \%$ de los padres manifestaron que el Personal de Enfermería les permitió la libre expresión de sus sentimientos y al mismo tiempo les animó a tener fe y esperanza en cuanto a la recuperación de sus hijos. Otros resultados reflejan que $83.33 \%$ de los padres manifestaron que este personal respetó su condición social en un alto porcentaje. De igual manera, los padres opinaron que el mismo personal respetó sus creencias y costumbres. Sin embargo, se encontraron respuestas preocupantes, como que el $60 \%$ de la población estudiada consideraron que el Personal de Enfermería no es cálido ni accesible, pero el 19, $66,66 \%$ consideran al Personal de Enfermería como profesionales dignos de confianza y respeto. 


\section{DISCUSIÓN}

Los resultados obtenidos permiten reflexionar sobre algunos aspectos que contrastan con lo establecido en otras investigaciones en la cuales se refleja la importancia de establecer la comunicación desde el mismo momento en que ingresa el recién nacido a la unidad, lo cual permitirá disminuir la ansiedad y el temor en los padres, además de generar confianza en el equipo de salud ${ }^{(11)}$. Cuando se observa en los resultados una tendencia a no informar sobre aspectos importantes sobre la terapéutica recibida por su hijo o hija, se reflejan diferencias en recomendaciones tales como la necesaria preparación a los padres para el primer encuentro con sus hijos, poniéndolos al tanto de las condiciones de la unidad y de los cuidados que están recibiendo en esos momentos, para facilitar su acercamiento e identificación y garantizar el inicio del vínculo afectivo entre los padres y el recién nacido ${ }^{(12)}$, lo cual implica una necesaria revisión sobre la comunicación como un proceso interactivo y relevante, mediante el cual se manifieste una clara intención de ayuda y se considere toda forma expresiva del profesional como fundamental en la satisfacción de las necesidades humanas.

Por otra parte, al analizar los resultados correspondientes al apoyo emocional se observan variaciones hacia opiniones positivas con respecto a la relación terapéutica. A pesar de que más de la mitad considera que el profesional de enfermería no demuestra afecto y, no es cálido y accesible, existe un alto porcentaje que valora el hecho de que los profesionales les proporcionen fe y esperanza, lo cual según Watson describe el papel de la enfermera(o) a la hora de desarrollar interacciones eficaces, además de incorporar valores humanísticos y altruistas, facilita la promoción del cuidado enfermero holístico y del cuidado positivo ${ }^{(2)}$. De allí que, al opinar en su mayoría que el profesional de enfermería se preocupa por cuidar a los recién nacidos y considerarlos como personal de confianza, concuerdan con otros estudios en los cuales se refleja la importancia que tiene para los padres en la recuperación de su bebé ${ }^{(13)}$.

\section{CONCLUSIÓN}

Aunque de manera general, estos datos reflejan una debilidad en la prestación de cuidados de enfermería de esta unidad a los padres de los neonatos hospitalizados, por lo que es necesario reforzar las actitudes y comportamientos positivos del profesional de enfermería hacia los padres, de manera que estos permitan el surgimiento de una actitud cálida, de respeto, comprensión y empatía, que les permita proporcionar un cuidado que ayude a los padres adaptarse a la unidad, y fomentar su capacidad de ser padres y madres de un recién nacido en condiciones de alto riesgo biológico.

El Personal de Enfermería que se encuentra en el área de la Unidad de Cuidados Intensivos Neonatales está llamado a fortalecer los aspectos positivos de la relación terapéutica con los padres de los neonatos hospitalizados en esta unidad, ayudando así a reconocer sus debilidades, a través de talleres de crecimiento personal que promuevan la empatía, la comunicación y cuidado de sí, para cuidar de los otros.

\section{BIBLIOGRAFÍA}

1. Satir V. Nuevas relaciones humanas en el núcleo familiar. 2da. Ed. México: Pax México; 2005. Tr: Rodríguez José

2. Marriner, A. Modelos y Teorías en Enfermería. 3ra. ed. Madrid: Mosby Doyma; 1995. 
3. Jofré R. Enríquez D. Nivel de estrés de las Madres con Recién Nacidos Hospitalizados en la UCIN Hospital Guillermo Grant. Ciencia y Enfermería, 2002; 8 (1): 31-36.

4. Amores S. Clínica del niño y su familia. Una perspectiva vincular psicoanalítica. Argentina: Distal; 2000. p.24

5. Bowlby J. Vinculas afectivos: formación, desarrollo y pérdida. 3 ed. Madrid: Ediciones Morata; 1999.

6. Klaus M, Kennell J. La relación madre hijo. Buenos Aires: Médica Panamericana; 1974.

7. Levin A. Viewpoint: Humane neonatal Care Initiative. Acta Paediatr 1999; 88: 353-5

8. Vieira GM. La Iniciativa de Humanización de Cuidados Neonatales. Rev. chil. pediatr. [online]. mar. 2003, vol.74, no.2 [citado 11 Febrero 2008], p.197-205. Disponible en la World Wide Web: <http://www.scielo. cl/scielo.php?script=sci_arttext\&pid=S0370$41062003000200009 \& \operatorname{lng}=$ es\&nrm=iso $>$. ISSN 0370-4106.

9. Lowdermilk DL, Perry SE, Bobak IM. Enfermería materno infantil. 6 ed. Barcelona, España: Harcourt/Océano; 1998

10. Parra FM. Moncada Z. Oviedo SJ. Marquina M. Estrés en padres de los recién nacidos hospitalizados en la Unidad de Alto Riesgo Neonatal. Index de Enfermería [Index Enferm] (edición digital) 2009; 18(1). [Consultado 20 Mayo 2009] Disponible en: http://www.indexf.com/index-enfermeria/v18n1/6804.php

11. Durán LN. Trujillo JM. Palmés QR. [online] Trabajo presentado en la modalidad de Poster en el XXI Congreso de la Asociación Nacional de Enfermería en Cuidados Intensivos y Pediátricos y Neonatales, La Palmas, España. Mayo 2000 [Consultado 15 Febrero 2008] Disponible en: https://secure.anecipn.org/congresos/archivo/XXI/html/ m1p6.htm

12. Arenas YL, Salgado CY, Eslava GD. Vivencia de los padres de niños hospitalizados en la unidad de recién nacidos de dos instituciones de salud de la ciudad de Bogotá. Enfermería Actual [online] 2006 ene vol. 8, $\mathrm{n}^{\circ} 2$ [citado 15 Enero 2008] 8(2): 8-13. Disponible en: URL: http://bases.bireme.br/ cgibin/wxislind.exe/iah/online/?IsisScript= iah/iah. xis\&base=LILACS\&lang=e\&nextAction=Ink\&isisFrom=1\&count=10\&exprSearch= Vivencias\%20and\%20padres\% 20and\%20recien\%20and\%20nacido

13. Sales S. Barbosa N. Henriquez J. (2004). Concepcoes das maes ao verem os filos hascerem prematuros e permanecerme em uma UTI Neonatal. Brasil. Resumen de presentado en IX Coloquio Panamericano de Investigación en Enfermería: Trabajos libres (6.224).Lima: OMS, OPS, Universidad Peruana Cayetano Heredia. 